\title{
StrokeBase: A Database of Cerebrovascular Disease-related Candidate Genes
}

\author{
Young-Uk Kim ${ }^{1 *}$, II-Hyun Kim, Ok-Sun Bang ${ }^{2}$ \\ and Young-Joo Kim ${ }^{1}$ \\ ${ }^{1}$ Medical Genomics Research Center, KRIBB, Daejeon \\ 305-806, Korea, ${ }^{2}$ Department of Medical Research, \\ KIOM, Daejeon 305-810, Korea
}

\begin{abstract}
Complex diseases such as stroke and cancer have two or more genetic loci and are affected by environmental factors that contribute to the diseases. Due to the complex characteristics of these diseases, identifying candidate genes requires a system-level analysis of the following: gene ontology, pathway, and interactions. A database and user interface, termed StrokeBase, was developed; StrokeBase provides queries that search for pathways, candidate genes, candidate SNPs, and gene networks. The database was developed by using in silico data mining of HGNC, ENSEMBL, STRING, RefSeq, UCSC, GO, HPRD, KEGG, GAD, and OMIM. Forty candidate genes that are associated with cerebrovascular disease were selected by human experts and public databases. The networked cerebrovascular disease gene maps also were developed; these maps describe genegene interactions and biological pathways. We identified 1127 genes, related indirectly to cerebrovascular disease but directly to the etiology of cerebrovascular disease. We found that a protein-protein interaction (PPI) network that was associated with cerebrovascular disease follows the power-law degree distribution that is evident in other biological networks. Not only was in silico data mining utilized, but also $250 \mathrm{~K}$ Affymetrix SNP chips were utilized in the 320 control/disease association study to generate associated markers that were pertinent to the cerebrovascular disease as a genome-wide search. The associated genes and the genes that were retrieved from the in silico data mining system were compared and analyzed. We developed a well-curated cerebrovascular disease-associated gene network and provided bioinformatic resources to cerebrovascular disease researchers. This cerebrovascular disease network can be used as a frame of systematic genomic research, applicable to other complex diseases. Therefore,
\end{abstract}

*Corresponding author: E-mail yjkim8@kribb,re.kr

Tel +82-42-879-8127, Fax +82-42-879-8119

Accepted 9 September 2008 the ongoing database efficiently supports medical and genetic research in order to overcome cerebrovascular disease.

Availability: StrokeBase is freely available at http:// sysbio.kribb.re.kr:8080/stroke/.

Keywords: stroke, cerebrovascular disease, SNP, disease gene, association study

\section{Introduction}

Cerebrovascular disease is the third-leading cause of death, behind heart disease and cancer (Toole. 1999). Complex disorders such as stroke and cancer have two or more genetic loci and environmental factors that contribute to the diseases (Braunwald et al., 2001; Jin et al., 2008). The objective of this study is to provide a systematic approach to analyze the complex effects of genes and to represent the frame of their relationships with specific diseases. To discover candidate genes that are related to complex disease, open sources, such as Entrez Pubmed, OMIM, PathwayStudio, HPRD, and Gencards, were thoroughly utilized, resulting in a vast amount of genetic and pathway information with regard to the etiology of cerebrovascular disease. These genes were annotated to access information on transcription, translation, structural function, and relatedness to the disease. In addition to in silico data mining, 320 250K Affymetrix SNP chips (GeneChip ${ }^{\text {TM }}$ Human Mapping 250K Nsp Array, Affymetrix, Inc., CA) were utilized for a case/control association study to generate experimentally associated markers of cerebrovascular disease. The associated genes from the SNP chips and the genes that were retrieved from in silico data mining systems were compared and analyzed. A protein-protein network diagram that showed the integrated markers and their relationships was constructed in order to analyze the network characteristics and produce hub genes. It was found that the PPI network that was associated with cerebrovascular disease follows a power-law degree distribution, as other biological networks do (Peri et al., 2003). The PathwayStudio 5.0 program (Ariadne, Inc., MD, USA) was utilized to process the natural text mining of PubMed abstracts; the use of PathwayStudio resulted in a gene-disease association network. The etiology of the disease and its related genes, which were extracted from in silico data mining and network analy- 
sis as well as from genome-wide association studies, were accumulated to the web-based portal database named StrokeBase (http://sysbio.kribb.re.kr:8080/stroke/) (Fig. 1). Information in this integrated cerebrovascular disease network may serve as a frame for systematic genomic research for cerebrovascular diseases, as well as for other complex diseases such as cancers.

\section{Utility and Discussion}

In order to map SNPs to disease-related genes, the chromosomal position of gene boundaries was retrieved from KnownGene, a program that provides information on protein-coding genes based on proteins from Uniprot with their corresponding mRNAs from GenBank. To link KnownGenes with gene symbols from OMIM and GAD, UCSC's table kgXref ("known genes to external reference") was used.

A database was developed by in silico data mining using HGNC, ENSEMBL, STRING, RefSeq, UCSC, GO, HPRD, KEGG, GAD, and OMIM (Fig. 1B). The Tomcat web server was utilized in StrokeBase, which simplifies building user interfaces for JSP (Java Server Page). Genes that were noted in Morbid Map (OMIM MorbidMap; http://www.ncbi.nlm.nih.gov/Omim/getmorbid.cgi) and the Genetic Association Database (GAD; http:// geneticassociationdb.nih.gov/) were selected (Hamosh et al., 2002). To cover up-to-date experimental results, a literature search also was performed by biologists. Because genes are noted by their various aliases, we mapped the gene set to the HUGO Gene Nomenclature Committee (HGNC, http://www.genenames.org/) to retain consistency of gene entries. We identified 1127 genes that were indirectly related to cerebrovascular disease but directly related to the etiology of cerebrovascular disease. The Pathway overview page has the list of signaling pathways that are related to cerebrovascular disease. This information refers to the pathway made by KEGG (Kyoto Encyclopedia of Genes and Genomes) (Kanehisa et al., 2000).

The cerebrovascular disease etiology map page refers to the risk factors that are associated with cerebrovascular disease and to the network of risk factors (Fig. 1C). If one of the risk factors is clicked, a gene list that is associated with the risk factor will be displayed. Forty genes that are associated with cerebrovascular disease were selected by human experts who utilized public databases (Fig. 1D).

250K Affymetrix SNP chips were utilized in the 320 control/disease association study in order to generate associated markers for cerebrovascular disease in a genome-wide search. Some of the candidate genes that
(A)

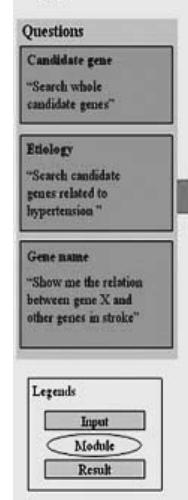

(C)
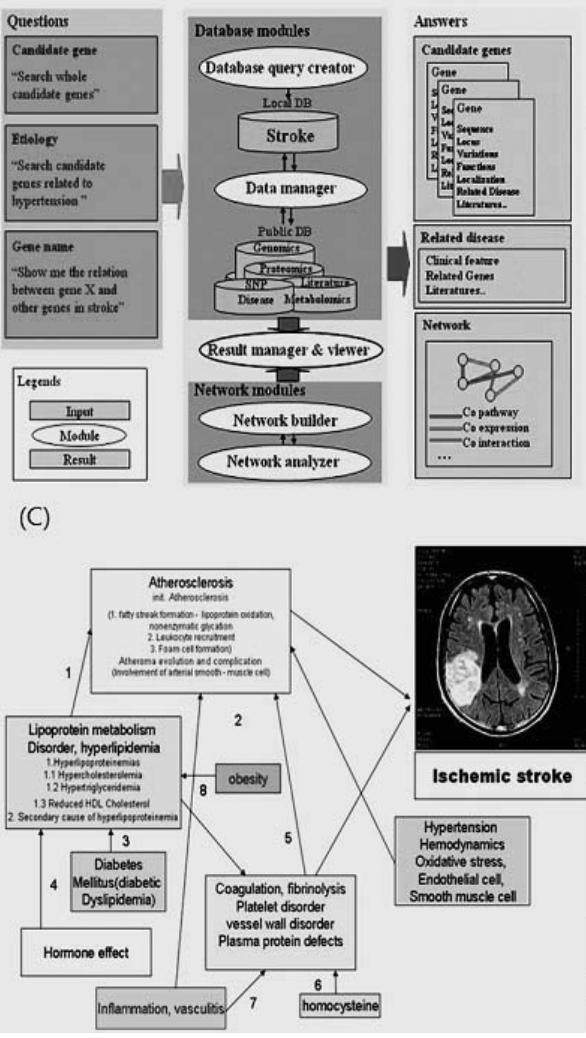

(B)

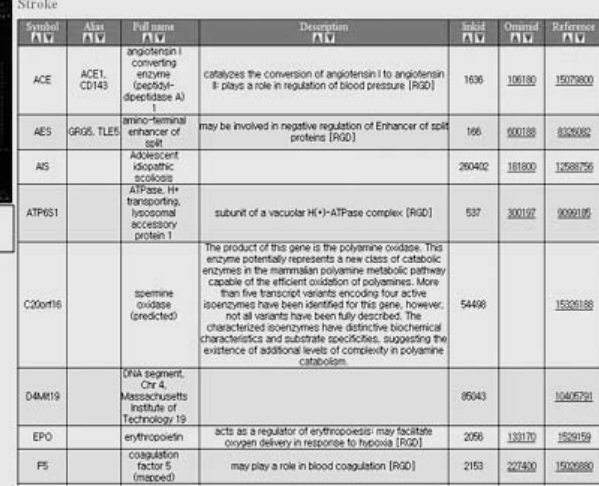

Fig. 1. Screenshots for StrokeBase (http://sysbio,kribb, re kr:8080/ stroke/). (A) The system structure for data manager, result viewer, and network analyzer. (B) The physical schema of the database. (C) Etiology map summarizing cerebrovascular disease risk factors. (D) Gene list associated with risk factors of cerebrovascular disease. Kim et al. 
(A)

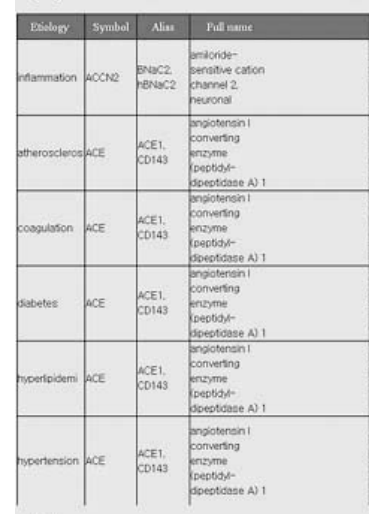

(C)

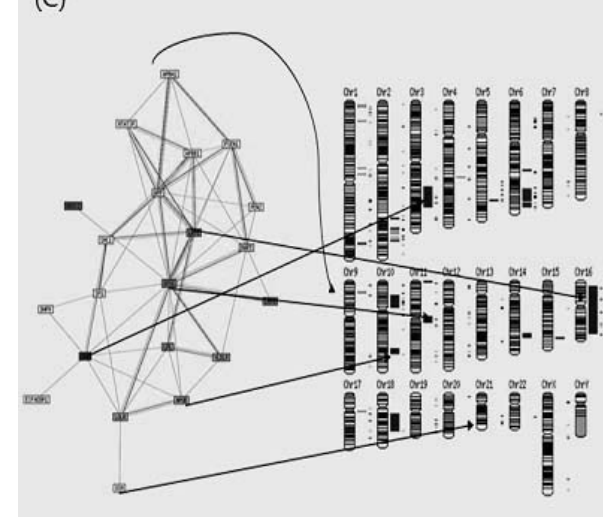

(B)

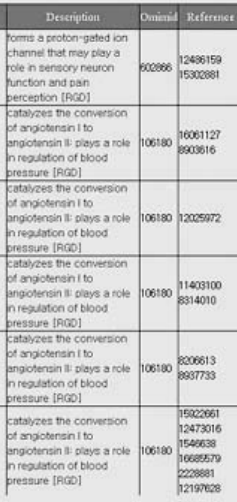

(D)
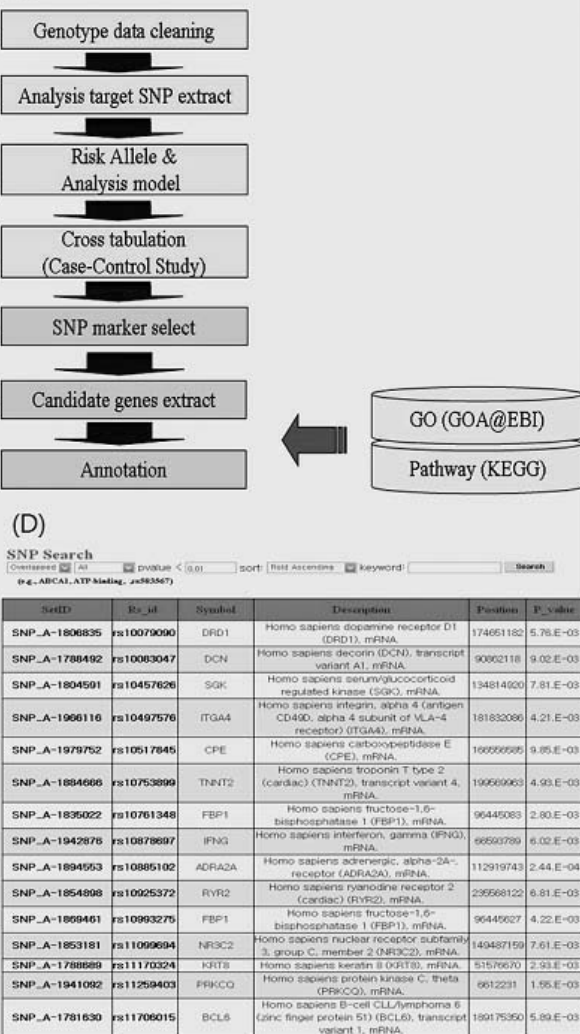

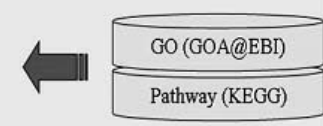

Fig. 2. Screenshots for the candidate genes identified in StrokeBase. (A) Search result table showing etiology candidate genes. (B) SNP chip data analysis flow. (C) Candidate gene network with chromosome mapping. (D) Search table showing associated SNPs by 320 250K Affymetrix SNP chips. Kim et al.

were identified in the chip analyses are shown (Fig. 2A). The SNP chip data analysis flow is shown (Fig. 2B). SNP-markers that were selected by the experiments were annotated and chromosomally mapped to reveal a comprehensive, networked gene map (Fig. 2C). We found overlaps between the gene markers that were annotated by experts and by the SNP experiment. Each SNP ID is linked to more detailed information. Selected SNPs with a given $p$-value are available in StrokeBase (Fig. 2D).

\section{Conclusion}

StrokeBase is an online database for cerebrovascular disease researchers. StrokeBase makes it possible for scientists to provide cerebrovascular disease association markers by in silico and whole-genome SNP arrays. Using data and tools that currently are available in StrokeBase, a scientist is able to analyze the complex effect of genes and to represent the frame of their relationships associated with cerebrovascular disease. The etiology of cerebrovascular disease was constructed, and 40 genes directly related to cerebrovascular disease were identified. Also, 1127 genes that were indirectly related to cerebrovascular disease but directly related to the etiology of cerebrovascular disease were identified.
The SNP chip-associated genes and those genes that were retrieved from in silico and data mining systems were compared. We developed a well-curated cerebrovascular disease-associated gene network and provided bioinformatic resources to cerebrovascular disease researchers. StrokeBase will thus serve as a novel and powerful resource for reviewing and interpreting cerebrovascular disease association data.

\section{Acknowledgments}

This research was supported by the Cerebrovascular Disease Project of the Ministry of Education, Science and Technology (MEST) and in part by the KRIBB Intramural Research Program.

\section{References}

Braunwald, E., Fauci, A.S., Kasper, D.L., Hauser, S.L., Longo, D.L., and Jameson, J.L. (2001). Harrison's principles of internal medicine (15th int. ed), McGraw-Hill.

Hamosh, A., Scott, A.F., Amberger, J., Bocchini, C., Valle, D., and McKusick, V.A. (2002). Online Mendelian Inheritance in Man (OMIM), a knowledgebase of human genes and genetic disorders. Nucleic Acids Res. 30, 52-55.

Jin, H., Kim, S.H., Kim, Y.U., Park, Y.K., Ji, M., and Kim, 
156 Genomics \& Informatics Vol. 6(3) 153-156, September 2008

Y.J. (2008). Development of KHapmap Browser using DAS for Korean HapMap Research. Genomics \& Informatics 6, 57-63.

Kanehisa, M., and Goto, S. (2000). KEGG: Kyoto Encyclopedia of Genes and Genomes. Nucleic Acids Res. 28, 27-30.

Peri, S. et al. (2003). Development of human protein reference database as an initial platform for approaching systems biology in humans. Genome Research 13, 23632371.

Toole, J.F. (1999). Chapter 13: Strokes in the young. Cerebrovascular disorders. Edition 5, Lippincott Williams \& Wilkins, Philadelphia, pp.283-316.

\section{Websites}

Becker, K.G., Barnes, K.C., Bright, T.J., and Wang, S.A. (2004). The Genetic Association Database. http:// geneticassociationdb.nih.gov/.

Kent, W.J., Sugnet, C.W., Furey, T.S., Roskin, K.M., Pringle, T.H., Zahler, A.M., and Haussler, D. (2002). The human genome browser at UCSC. http://genome.ucsc.edu/.

McKusick-Nathans Institute for Genetic Medicine, Johns Hopkins University (Baltimore, MD) and National Center for Biotechnology Information, National Library of Medicine (Bethesda, MD). (2000). Online Mendelian Inheritance in Man, OMIM (TM). http://www.ncbi.n/m.nih.gov/omim/.

Nucleus Medical Art: 3D Medical Animations. (2008). http://Www.nucleusinc.com.

Sherry, S.T., Ward, M.H., Kholodov, M., Baker, J., Phan, L., Smigielski, E.M., and Sirotkin, K. (2001). dbSNP: the NCBI database of genetic variation. http://Www.ncbi,nlm.nih. gov/SNP/.

xPharm: The Comprehensive Pharmacology Reference. (2008). http://www.xpharm,com/citation?Article_ID=1050. 